\title{
Assessment of Reliability of Cone-beam Computed Tomography in Skeletal and Dental Discrepancy
}

\author{
Ashok Khandelwal ${ }^{1}$, Shweta Raghav ${ }^{2}$, Kamalshikha Baheti ${ }^{3}$, Anurag Jindal ${ }^{4}$, Shubhanjali Bansod ${ }^{5}$, Amit Singh Choudhary ${ }^{6}$
}

\begin{abstract}
Aim: The present study was conducted to evaluate the reliability of cone-beam computed tomography (CBCT) in dental asymmetry with skeletal midline deviation.

Materials and methods: This study was conducted on 124 patients (males 52, females 72) who underwent orthodontic treatment. The CBCT scan was obtained with the Planmica machine. Three maxillofacial radiologists evaluated 10 landmarks and 12 distances on maxillary and mandibular arches on CBCT images in all patients.

Results: Interobserver mean difference at point 1 was 0.33 ; point 2, 0.34; point 3, 0.32; point 4, 0.58; point 5, 0.56; point 6, 0.44; point 7, 0.28; point $8,0.22$; point 9, 0.54 ; point $10,0.21$; point $11,0.34$; and point $12,0.36$. The discrepancy between intra- and interobserver was not considerable $(p>0.05)$.

Conclusion: Cone-beam computed tomography is useful for diagnosis of skeletal asymmetry.

Clinical significance: Cone-beam computed tomography is a reliable and reproducible tool for diagnosis of skeletal asymmetry for successful orthodontic treatment.

Keywords: Asymmetry, Cone-beam computed tomography, Malocclusion.

The Journal of Contemporary Dental Practice (2020): 10.5005/jp-journals-10024-2844
\end{abstract}

\section{INTRODUCTION}

Malocclusion is the leading cause of unaesthetic appearance for which the patient visit the dentist to seek advice. Malocclusion among population is matter of concern as teeth are the first that are exposed when we speak. Facial asymmetry and dental midlines are variable features that are not coinciding with each other. Angle has classified malocclusion into class I, class II, and class III malocclusion. ${ }^{1}$ There are further modifications in Angle's classification. Class II div $I$ is considered when the distobuccal cusp of the maxillary first molar coinsides with the mesiobuccal groove of the mandibular first molar. There is increased overjet in class II division I malocclusion. In class II div II malocclusion, there is retroclined maxillary central incisors leading to decreased overjet. Dewey's classification of malocclusion also provides useful information. To correct dental arch rotation, extractions is required to correct a dental midline shift. ${ }^{2}$ Thus, skeletal and dental discrepancy has to be corrected for the management of malocclusion. To diagnose patient's dental symmetry in association with skeletal midline deviation in the midsagittal plane, various methods have been employed. ${ }^{3}$

Bolt suggested that symmetric grid or bow divider with ruler can be used in identifying asymmetry. ${ }^{4}$ Several authors have experimented scan images of plaster models with the help of computers. Posteroanterior (PA) radiography skull and mandible, as recommended by Burstone, is one of the methods to calculate divergence in the maxilla and mandible along with the midline of the upper and lower dental aspect to skeletal one. ${ }^{5}$ But this technique has its limitations. Considering, median raphe as the patient's skeletal midline, the dental midline can be evaluated. Studies suggested the role of dental casts in establishing the relationship between teeth and bone. ${ }^{6}$ Cone-beam computed tomography $(\mathrm{CBCT})$ is one of the recent diagnostic aids in assessing skeletal and dental discrepancy. The present study was conducted \begin{tabular}{l}
\hline \hline${ }^{1-6}$ Department of Orthodontics and Dentofacial Orthopedics, College \\
of Dental Science and Hospital, Rau, Indore, Madhya Pradesh, India \\
Corresponding Author: Shweta Raghav, Department of Orthodontics \\
and Dentofacial Orthopedics, College of Dental Science and Hospital, \\
Rau, Indore, Madhya Pradesh, India, Phone: +91 9669854055, e-mail: \\
shwetaraghav4u1@gmail.com
\end{tabular}

How to cite this article: Khandelwal A, Raghav S, Baheti K, et al. Assessment of Reliability of Cone-beam Computed Tomography in Skeletal and Dental Discrepancy. J Contemp Dent Pract 2020;21(7): 765-768.

Source of support: Nil

Conflict of interest: None

to evaluate the reliability of $\mathrm{CBCT}$ in dental asymmetry with skeletal midline deviation.

\section{Materials and Methods}

This study was conducted in the Department of Orthodontics and Dentofacial Orthopedics, College of Dental Science and Hospital, Rau, Indore on 124 patients (males 52, females 72) who underwent orthodontic treatment. An ethical approval about the study was obtained from the institutional ethics committee prior to the study. All participants' informed approval was attained prior to the study.

The inclusion criteria were patient's age ranged $14-25$ years and those who willingly participated in the study. The exclusion criteria were patients with missing anterior and molars and patients with cleft and palate.

All participants underwent $\mathrm{CBCT}$ scan taken with the Planmica machine operating at $140 \mathrm{kVp}$ and $10 \mathrm{~mA}$. The $1 \mathrm{~mm}$ slices were used in the study. The Frankfort horizontal plane was oriented horizontally and the skeletal midline was compared with the sagittal

() The Author(s). 2020 Open Access This article is distributed under the terms of the Creative Commons Attribution 4.0 International License (https://creativecommons. org/licenses/by-nc/4.0/), which permits unrestricted use, distribution, and non-commercial reproduction in any medium, provided you give appropriate credit to the original author(s) and the source, provide a link to the Creative Commons license, and indicate if changes were made. The Creative Commons Public Domain Dedication waiver (http://creativecommons.org/publicdomain/zero/1.0/) applies to the data made available in this article, unless otherwise stated. 


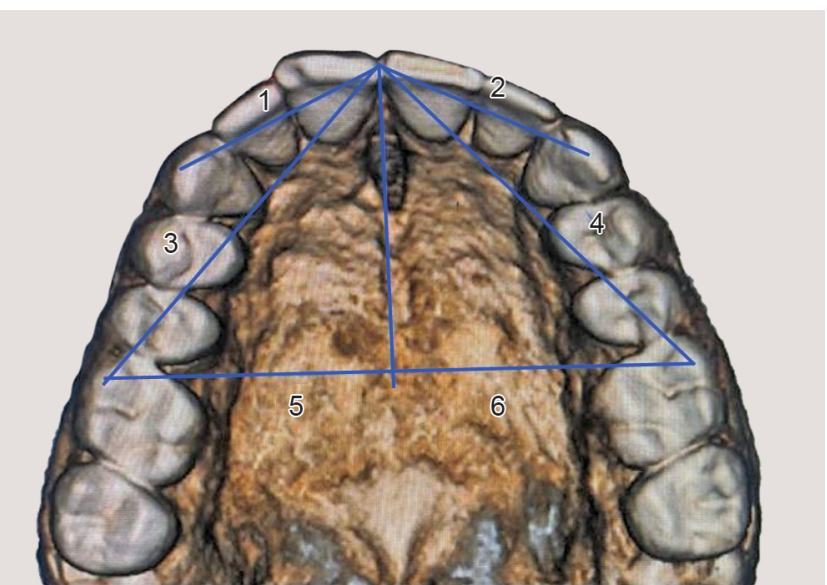

Fig. 1: Maxillary landmarks and distances

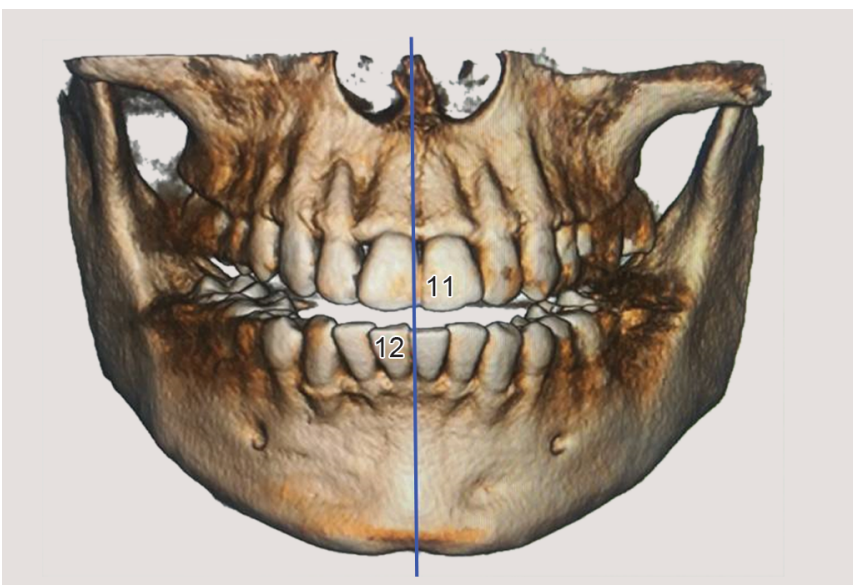

Fig. 3: Landmarks in midline

plane. Three maxillofacial radiologists evaluated 10 landmarks (5 maxillary and 5 mandibular) and 12 distances (6 maxillary, 4 mandibular, and 2 midline) on maxillary and mandibular arches on CBCT images in all patients (Figs 1 to 3). Landmarks were plotted on the axial view of multiplanar reconstruction (MPR). All the investigation was done by three trained investigators. To overcome intraobserver observations, each observer recorded three values for each landmark at different time and their mean was taken as the final value.

Results were tabulated and statistically evaluated by SPSS IBM version 21 using intraclass correlation coefficients (ICCS) and compared using Chi-square and $t$ tests. The $p$ value was believed considerable at lower than 0.05 .

\section{Results}

Table 1 shows five maxillary and five mandibular landmarks used for the study. Table 2 shows six maxillary, four mandibular, and two midline distances measured in the study (Figs 1 to 3). Table 3 shows intraobserver and interobserver reliability measured by ICC for the distances measured. The discrepancy between intra- and interobserver was not considerable $(p>0.05)$.

Figure 4 shows intraobserver mean difference of distance measured between the landmarks as 12 points. At point 1 was 0.33 ; point $2,0.34$; point $3,0.32$; point $4,0.58$; point $5,0.56$; point 6 , 0.44 ; point $7,0.28$; point $8,0.22$; point $9,0.54$; point $10,0.21$; point

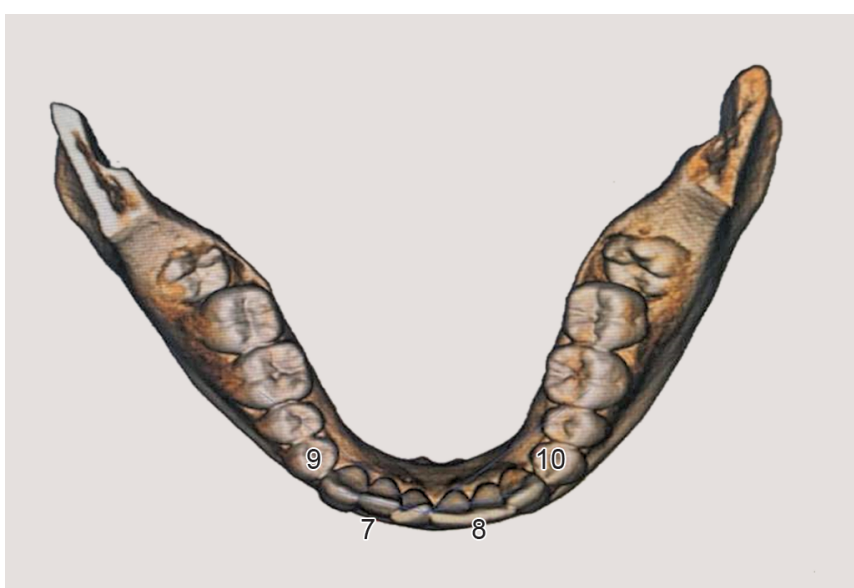

Fig. 2: Mandibular landmarks and distances

Table 1: Landmarks used in the study

\begin{tabular}{ll}
\hline Symbols & Description \\
\hline Uaxillary landmark & \\
UR3 & Right molar mesiobuccal cusp tip \\
UML & Right canine cusp tip \\
UL6 & Skeletal midline at upper incisors incisor \\
UL3 & edge \\
Mandibular landmark & Left molar mesiobuccal cusp tip \\
LR6 & Left canine cusp tip \\
LR3 & Right molar mesiobuccal cusp tip \\
LML & Right canine cusp tip \\
& Skeletal midline at lower incisors incisor \\
LL6 & edge \\
LL3 & Left molar mesiobuccal cusp tip \\
\hline
\end{tabular}

Table 2: Measurements used in the study

\begin{tabular}{cl}
\hline Distance & Description \\
\hline Maxillary measurements & \\
1 & Distance between UR3 and UML \\
2 & Distance between UL3 and UML \\
3 & Distance between UR6 and UML \\
4 & Distance between UL6 and UML \\
5 & Distance between UR6 $90^{\circ}$ and the \\
& skeletal midline \\
6 & Distance between UL6 $90^{\circ}$ and the \\
Mandibular measurements & skeletal midline \\
7 & Distance between LR3 and LML \\
8 & Distance between LL3 and LML \\
9 & Distance between LR6 and LML \\
10 & Distance between LL6 and LML \\
Midline & \\
11 & Distance between skeletal midline \\
12 & and midline of upper teeth \\
& Distance between skeletal midline \\
& and midline of lower teeth \\
\hline
\end{tabular}


$11,0.34$; and point $12,0.36$. Figure 5 shows interobserver difference at 12 points, which was nonsignificant. The discrepancy between intra- and interobserver was not considerable $(p>0.05)$.

\section{Discussion}

Malocclusion is the leading dental complaint for which patients visit a dentist. It is quite common among youngsters. Dental asymmetry is the biggest problem most of the people have encountered. Careful evaluation of anatomical landmarks and points may be useful in assessment of malocclusion in patients. There have been numerous methods proposed in the past., ${ }^{7,8}$ This study was conducted to assess the asymmetry in relation to canine, molars, and dental midline in concern to the skeletal midline using CBCT. We included 124 patients with malocclusion. In all patients, the CBCT scan was obtained with the Planmica machine. The exposure parameters such as $\mathrm{kVp}$ and $\mathrm{mA}$ were kept constant in all patients.

We observed that five landmarks in maxilla and five in mandibles were utilized. These landmarks have been studied in the previous studies also and are capable of providing dental and skeletal discrepancy.

It consisted of right molar mesiobuccal cusp tip (UR6), right canine cusp tip (UR3), skeletal midline at upper incisors incisor edge

Table 3: Intra- and interobserver reliability measured by ICC for the measured distances

\begin{tabular}{lcccc}
\hline & \multicolumn{2}{c}{ Intraobserver } & \multicolumn{2}{c}{ Interobserver } \\
\cline { 2 - 5 } Values & Number & Percentage & Number & Percentage \\
\hline$>0.90$ & 62 & 50 & 60 & 48.4 \\
$0.75-0.90$ & 43 & 34.6 & 42 & 33.8 \\
$0.45-0.75$ & 19 & 15.4 & 22 & 17.8 \\
$<0.45$ & 0 & 0 & 0 & 0 \\
Total & 124 & 100 & 124 & 100 \\
\hline
\end{tabular}

$p>0.05$

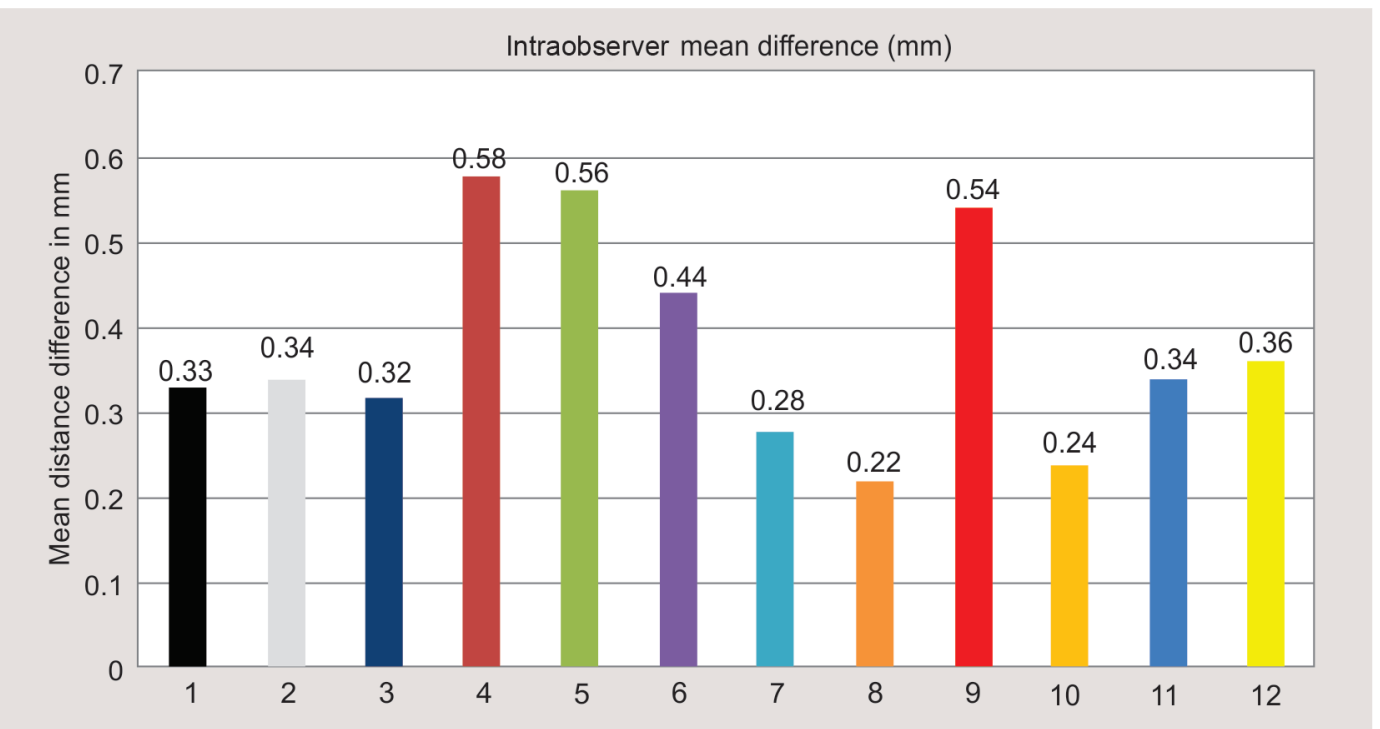

Fig. 4: Reliability measured by intraclass ICC for each distance

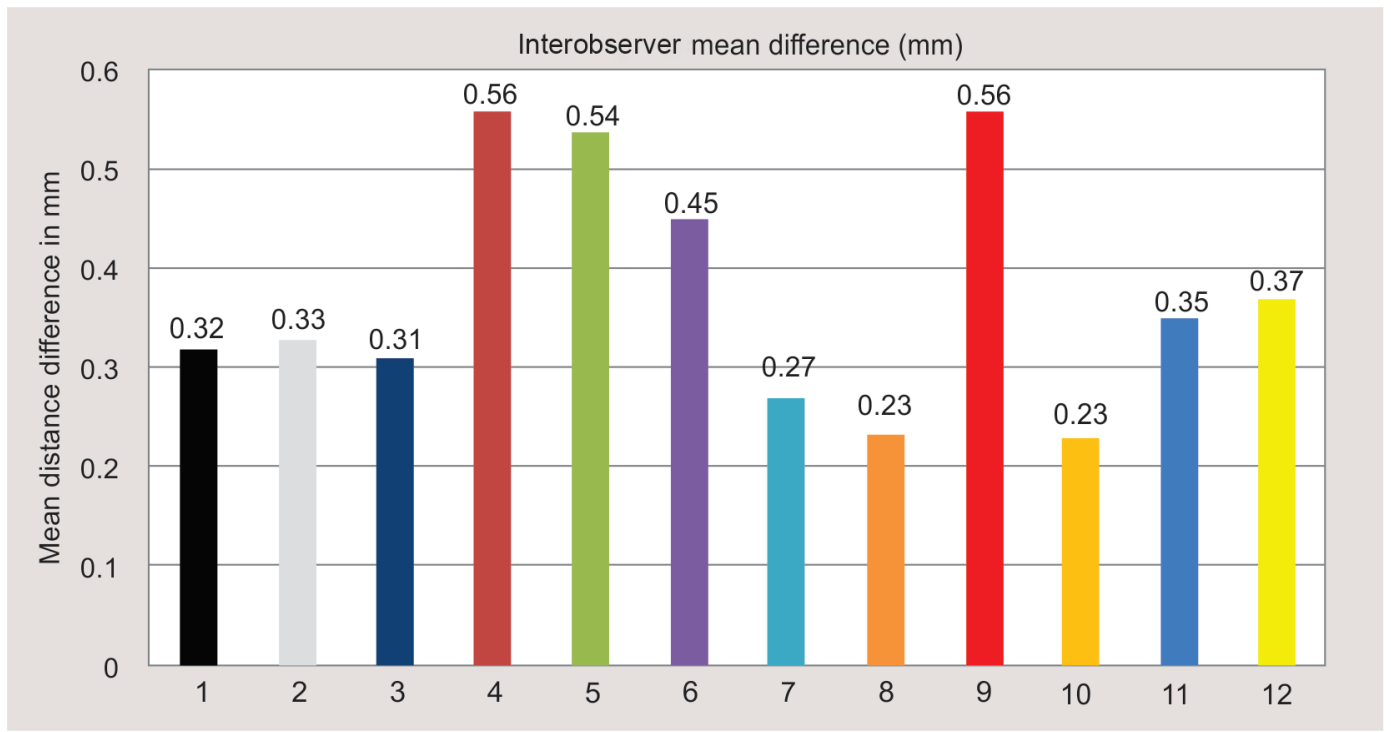

Fig. 5: Reliability measured by interclass ICC for each distance 
(UML), left molar mesiobuccal cusp tip (UL6), left canine cusp tip (UL3) and five in mandible right molar mesiobuccal cusp tip (LR6), right canine cusp tip (LR3), skeletal midline at lower incisors incisor edge (LML), left molar mesiobuccal cusp tip (LL6), and left canine cusp tip (LL3).

Sanders et al. in their study utilized Dolphin 3D in evaluation parameters in relation to dental, cranial base, maxillary, and mandibular teeth. A total of 34 anatomical landmarks were evaluated with $C B C T$ scans. Using $3 D$, all reference points were digitized and assessed by means of dental and skeletal dimensions. The study revealed slightly longer length of dental arches on the left side than the right side. There was slight dental midline discrepancy. ${ }^{9}$

We assessed six distances in the maxilla, four in the mandible, and two in the midline. It was distance between the right canine cusp tip and the skeletal midline at the upper incisors incisor edge, distance between the left canine cusp tip and the skeletal midline at the upper incisors incisor edge, distance between the right molar mesiobuccal cusp tip and the skeletal midline at the upper incisors incisor edge, distance between the left molar mesiobuccal cusp tip and the skeletal midline at the upper incisors incisor edge, distance between the right molar mesiobuccal cusp tip $90^{\circ}$ and the skeletal midline, distance between the left molar mesiobuccal cusp tip $90^{\circ}$ and the skeletal midline, distance between the right canine cusp tip and the skeletal midline at the lower incisors incisor edge, distance between the left canine cusp tip and the skeletal midline at the lower incisors incisor edge, distance between the right molar mesiobuccal cusp tip and the skeletal midline at the lower incisors incisor edge, distance between the left molar mesiobuccal cusp tip and the skeletal midline at the lower incisors incisor edge, distance between the skeletal midline and the midline of upper teeth, and distance between the skeletal midline and the midline of lower teeth.

Ruellas et al. in their study assessed dental asymmetry in relation to the skeleton midline. For the study, landmarks and distances were evaluated using CBCT. Authors observed $\geq 0.9$ in $90 \%$ subjects and $83 \%$ variables for intra- and interobserver, respectively. It was found that ICC for distances was $\geq 0.9$ for 58 and $42 \%$, respectively. ${ }^{10}$ In our study, intraobserver and interobserver reliability measured by ICC for the distances measured, which was nonsignificant $(p>0.05)$. Ludlow et al. in his study found intraexaminer mean differences for variable as $\leq 0.05 \mathrm{~mm}$ on dental casts and $\leq 0.32 \mathrm{~mm}$ on 3D digital study models. ${ }^{11}$

We measured mean difference for distances from skeletal to dental midlines and it was found to be $\leq 0.4 \mathrm{~mm}$. Good reliability between observers was observed with the molars perpendicular line to the skeletal midline. Palatal rugae, as a reference point used for conventional or 3D digital models, is consistent for growing patients. But due to different shapes and curvatures of palatal raphae, this cannot be considered as good choice for the skeletal midline.

Kapila et al. in his study measured landmarks on stack of slices instead of rendered images. In the present study, measurement was done on axial sections of MPR. Cone-beam computed tomography offers less patient radiation exposure and is relatively cheaper and accurate as compared to the CT scan. The radiation doses used in CBCT are comparable to full-mouth radiographs. It can provide multiple views of $0.1 \mathrm{~mm}$ thickness and has ability to show soft tissues, sinus, TMJ, etc. ${ }^{12}$

Almeida et al. conducted a study to assess skeletal and dental discrepancy using CBCT and found CBCT as one of useful tools in determining malocclusion. He further stated that $3 \mathrm{D}$ images are better indicative of malocclusion when it is compared with skeletal malocclusion. ${ }^{13}$ Abdelkarim et al. from his review stated that CBCT can be used as a diagnostic tool for various orthodontic treatments need. ${ }^{14}$ Ruellas et al. concluded form his study that CBCT is a valid method to assess skeletal asymmetry. ${ }^{3}$

Evaluation of orthodontic patients with CBCT images helps to rule out skeletal discrepancy for successful treatment outcome in practice of the primary care procedure. The limitation of the present study is the undersized sample range. Further studies are required to evaluate the validity of CBCT on a larger sample size.

\section{Conclusion}

Dental asymmetry can be assessed with CBCT images. Dental asymmetry can be effectively evaluated and asymmetry of incisors, molars, and canines in relation to skeletal midline may be efficiently determined with CBCT. This study helps to utilize $C B C T$ in evaluation of dental and skeletal discrepancy for effective management of malocclusion cases.

\section{References}

1. Agrawal M, Agrawal JA, Nanjannawar L, et al. Dentofacial asymmetries: challenging diagnosis and treatment planning. J Int Oral Health 2015;7(7):128-131.

2. Cevidanes LH, Oliveira AE, Grauer D, et al. Clinical application of $3 \mathrm{D}$ imaging for assessment of treatment outcomes. Semin Orthod 2014;17(1):72-80. DOI: 10.1053/j.sodo.2010.08.012.

3. Meloti AF, Gonçalves Rde C, Silva E, et al. Lateral cephalometric diagnosis of asymmetry in angle class II subdivision compared to class I and II. Dental Press J Orthod 2014;19(4):80-88. DOI: 10.1590/21769451.19.4.080-088.oar.

4. Boldt F, Weinzierl C, Hertrich K, et al. Comparison of the spatial landmark scatter of various 3D digitalization methods. J Orofac Orthop 2009;70(3):247-263. DOI: 10.1007/s00056-009-0902-2.

5. da Neiva MB, Soares ÁC, Lisboa Cde O, et al. Evaluation of cephalometric landmark identification on CBCT multiplanar and 3D reconstructions. Angle Orthod 2015;85(1):11-17. DOI: 10.2319/120413891.1.

6. Škrinjarić A, Mladen Šlaj M, Šlaj M. Fluctuating dental arch asymmetry in different malocclusion groups. Acta stomatol Croat 2018;52(2):105113. DOI: $10.15644 /$ asc52/2/3.

7. Pittayapat P, Limchaichana-Bolstad N, Willems G, et al. Threedimensional cephalometric analysis in orthodontics: a systematic review. Orthod Craniofac Res 2014;17(2):69-91. DOI: 10.1111/ocr.12034.

8. Lenza MA, de Carvalho AA, Lenza EB, et al. Radiographic evaluation of orthodontic treatment by means of four different cephalometric superimposition methods. Dental Press J Orthod 2015;20(3):29-36. DOI: 10.1590/2176-9451.20.3.029-036.oar.

9. Sanders AD, Chandhoke AK, Uribe FA, et al. Quantification of skeletal asymmetries in normal adolescents: cone-beam computed tomography analysis. Progress Orthod 2014;15(1):26. DOI: 10.1186/ s40510-014-0026-0.

10. Ruellas ACO, Koerich L, Baratieri C, et al. Reliability of CBCT in the diagnosis of dental asymmetry. Dental Press J Orthod 2014;19(2):90 95. DOI: 10.1590/2176-9451.19.2.090-095.oar.

11. Ludlow JB, Davies-Ludlow LE, Brooks SL. Dosimetry of two extraoral direct digital imaging devices: NewTom cone beam CT and Orthophos plus DS panoramic unit. Dentomaxillofac Radiol 2003;32(4):229-234. DOI: $10.1259 / \mathrm{dmfr} / 26310390$.

12. Kapila S, Conley RS, Harrell Jr WE. The current status of cone beam computed tomography imaging in orthodontics. Dentomaxillofac Radiol 2014;40(1):24-34. DOI: 10.1259/dmfr/12615645.

13. Almeida MA, Phillips C, Kula K, et al. Stability of the palatal rugae as landmarks for analysis of dental casts. Angle Orthod 2014;65(1):43-48.

14. Abdelkarim A. Cone-beam computed tomography in orthodontics. Dent J 2019;7(3):1-31. 